\title{
A new two-variable generalization of the chromatic polynomial
}

\author{
Klaus Dohmen, André Pönitz, Peter Tittmann \\ Department of Mathematics, Mittweida University of Applied Sciences, 09648 Mittweida, Germany \\ E-mail: dohmen@htwm.de, poenitz@htwm.de, peter@htwm.de
}

received July 31, 2002, revised February 20, 2003, May 15, 2003, June 12, 2003, accepted June 16, 2003.

Let $P(G ; x, y)$ be the number of vertex colorings $\phi: V \rightarrow\{1, \ldots, x\}$ of an undirected graph $G=(V, E)$ such that for all edges $\{u, v\} \in E$ the relations $\phi(u) \leq y$ and $\phi(v) \leq y$ imply $\phi(u) \neq \phi(v)$. We show that $P(G ; x, y)$ is a polynomial in $x$ and $y$ which is closely related to Stanley's chromatic symmetric function, and which simultaneously generalizes the chromatic polynomial, the independence polynomial, and the matching polynomial of $G$. We establish two general expressions for this new polynomial: one in terms of the broken circuit complex, and one in terms of the lattice of forbidden colorings. Finally, we give explicit expressions for the generalized chromatic polynomial of complete graphs, complete bipartite graphs, paths, and cycles, and show that $P(G ; x, y)$ can be evaluated in polynomial time for trees and graphs of restricted pathwidth.

Keywords: chromatic polynomial, set partition, broken circuit, pathwidth, chromatic symmetric function

\section{Introduction}

All graphs in this paper are assumed to be finite, undirected and without loops or multiple edges. We write $G=(V, E)$ to denote that $G$ is a graph having vertex set $V$ and edge set $E$.

The well-known chromatic polynomial $P(G ; y)$ of a graph $G=(V, E)$ gives the number of vertexcolorings of $G$ with at most $y$ colors such that adjacent vertices receive different colors. For an introduction to chromatic polynomials the reader is referred to READ [7].

TUTTE [13, 14] has generalized the chromatic polynomial to the Tutte polynomial $T(G ; x, y)$. We propose a different generalization of the chromatic polynomial by weakening the requirements for proper colorings: Let $x=\mathcal{Y} \cup Z, \mathcal{Y} \cap Z=\emptyset$, be the set of available colors with $|X|=x$ and $|\mathcal{Y}|=y$. Then, a generalized proper coloring of $G$ is a map $\phi: V \rightarrow X$ such that for all $\{u, v\} \in E$ with $\phi(u) \in \mathcal{Y}$ and $\phi(v) \in \mathcal{Y}$ the relation $\phi(u) \neq \phi(v)$ holds. Consequently, adjacent vertices can be colored alike only if the color is chosen from $Z$. In order to distinguish between these two sets of colors we call the colors of $\mathcal{Y}$ proper and the colors of $Z$ improper. The number of generalized proper colorings of $G$ is denoted by $P(G ; x, y)$, and as we will see in Theorem 11 below, this number turns out to be a polynomial in $x$ and $y$, which we refer to as the generalized chromatic polynomial of $G$. Despite its simple definition, this new two-variable polynomial shows some interesting properties which are discussed later on in more detail: 
- It generalizes the chromatic polynomial, the independence polynomial, and the matching polynomial.

- It is closely related to Stanley's chromatic symmetric function [11].

- It distinguishes all non-isomorphic trees with up to nine vertices.

- It satisfies both an edge decomposition formula and a vertex decomposition formula.

In subsequent sections, we give explicit expressions for the generalized chromatic polynomial of complete graphs, complete bipartite graphs, paths, and cycles, and show that our generalized chromatic polynomial can be evaluated in polynomial time for trees and graphs of restricted pathwidth.

\section{Basic properties}

Let $G=(V, E)$ be a graph, and let $n=|V|$ and $m=|E|$ denote the number of vertices and edges of $G$, respectively. By considering $\mathcal{Y}=\emptyset$ we get $P(G ; x, 0)=x^{n}$. The case $Z=\emptyset$ shows that $P(G ; y, y)$ coincides with the usual chromatic polynomial $P(G ; y)$. For any edge $e \in E$ let $G-e$ and $G / e$ be the graphs obtained from $G$ by deleting resp. contracting $e$ and then, in the resulting multigraph, replacing each class of parallel edges by a single edge. By the edge decomposition formula for the usual chromatic polynomial we have

$$
P(G ; y, y)=P(G-e ; y, y)-P(G / e ; y, y) .
$$

Since vertices belonging to different components of $G$ can be colored independently, we find that if $G_{1}, \ldots, G_{k}$ are the connected components of $G$, then

$$
P(G ; x, y)=\prod_{i=1}^{k} P\left(G_{i} ; x, y\right) .
$$

The following theorem shows that $P(G ; x, y)$ can be expressed in terms of chromatic polynomials of subgraphs of $G$. Let $G-X$ be the subgraph obtained from $G$ by removing all vertices of a vertex subset $X \subseteq V$. For short, we write $G-v$ instead of $G-\{v\}$.

Theorem 1 Let $G$ be a graph. Then,

$$
P(G ; x, y)=\sum_{X \subseteq V}(x-y)^{|X|} P(G-X ; y) .
$$

Proof. Every generalized proper coloring of $G$ can be obtained by first choosing a subset $X$ of $V$ that is colored with colors of $Z$. There are $(x-y)^{|X|}$ different colorings for these vertices. The remaining subgraph has to be colored properly using colors of $\mathcal{Y}$, for which there are $P(G-X ; y)$ possibilities.

Corollary 2 Let $a_{i}$ denote the number of independent vertex sets of cardinality $n-i$ where $n$ denotes the number of vertices of $G$. Then the independence polynomial of $G$, defined by $I(G, x):=\sum_{i=0}^{n} a_{i} x^{i}$, satisfies

$$
I(G, x)=P(G ; x+1,1) .
$$

In particular, $P(G ; 2,1)$ is the number of independent vertex sets of $G$. 
Proof. By Theorem 1 we have

$$
P(G ; x+1,1)=\sum_{X \subseteq V} x^{|X|} P(G-X ; 1)
$$

Note that the chromatic polynomial $P(G-X ; 1)$ equals 1 if $V-X$ is an independent set (a set of isolated vertices) of $G$; otherwise, $P(G-X ; 1)=0$. Consequently, the coefficient of $x^{i}$ in $P(G ; x+1,1)$ counts the independent sets of cardinality $n-i$ in $G$.

Theorem 3 The generalized chromatic polynomial $P(G ; x, y)$ satisfies

$$
\frac{\partial}{\partial x} P(G ; x, y)=\sum_{v \in V} P(G-v ; x, y) .
$$

Proof. From Theorem $\mathrm{W}$ it follows that

$$
\begin{aligned}
\sum_{v \in V} P(G-v ; x, y) & =\sum_{v \in V} \sum_{W \subseteq V \backslash\{v\}}(x-y)^{|W|} P(G-W-v ; y) \\
& =\sum_{X \subseteq V}|X|(x-y)^{|X|-1} P(G-X ; y) \\
& =\frac{\partial}{\partial x}\left(\sum_{X \subseteq V}(x-y)^{|X|} P(G-X ; y)\right) \\
& =\frac{\partial}{\partial x} P(G ; x, y)
\end{aligned}
$$

which gives the result.

We close this section by remarking that our generalized chromatic polynomial is not an evaluation of the Tutte polynomial [13, 14]: The Tutte polynomial $T(G ; x, y)$ equals $x^{n-1}$ for every tree having $n$ vertices regardless of the structure of the tree. However, there are non-isomorphic trees with different generalized chromatic polynomials. The smallest example for such a pair of trees is presented in Figure 1. The generalized chromatic polynomials of these two trees $P_{4}$ and $S_{3}$ are respectively given by

$$
\begin{aligned}
& P\left(P_{4} ; x, y\right)=x^{4}-3 x^{2} y+y^{2}+2 x y-y \\
& P\left(S_{3} ; x, y\right)=x^{4}-3 x^{2} y+3 x y-y
\end{aligned}
$$

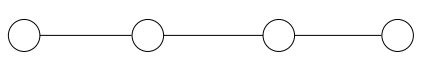

Fig. 1: Non-isomorphic trees $P_{4}$ and $S_{3}$ having four vertices and distinct generalized chromatic polynomials. 


\section{The partition representation of $P(G ; x, y)$}

Let $G=(V, E)$ be a graph and $\Pi(V)$ be the partition lattice of the vertex set $V$. A subset $W \subseteq V$ is connected if the subgraph of $G$ induced by the vertices of $W$ is connected. Let $\Pi_{G}$ be the set of all partitions $\pi$ of $V$ such that every block of $\pi$ is connected. Then $\Pi_{G}$ forms a geometric sublattice of $\Pi(V)$ (see e.g. [10]). The unique minimal element $\hat{0}$ in $\Pi_{G}$ is the finest partition consisting only of singletons. We show that $\Pi_{G}$ corresponds to the lattice of forbidden colorings of $G$. Let $f(\pi)$ be the number of colorings of $V$ with $y$ proper colors and $x-y$ improper colors such that

1. the vertices of each block $B$ of $\pi$ all get the same color,

2. if $|B|=1$ then that color can be any color of $\mathcal{X}$ while if $|B| \geq 2$ then that color must be from $\mathcal{Y}$, and

3. no two blocks which are connected by an edge may be assigned the same color of $\mathcal{Y}$.

Consequently, no proper coloring of $G$ contributes to $f(\pi)$ for $\pi>\hat{0}$. We denote by $|\pi|$ the number of blocks of $\pi$. Let $k_{1}(\pi)$ be the number of singletons of $\pi$. We obtain

$$
x^{k_{1}(\pi)} y^{|\pi|-k_{1}(\pi)}=\sum_{\substack{\sigma \in \in_{G} \\ \sigma \geq \pi}} f(\sigma) .
$$

Our generalized chromatic polynomial $P(G ; x, y)$ corresponds to $f(\hat{0})$. Thus, by Möbius inversion we obtain the following theorem.

Theorem 4 The generalized chromatic polynomial can be expressed as a sum over the lattice $\Pi_{G}$ :

$$
P(G ; x, y)=\sum_{\pi \in \Pi_{G}} \mu(\hat{0}, \pi) x^{k_{1}(\pi)} y^{|\pi|-k_{1}(\pi)} .
$$

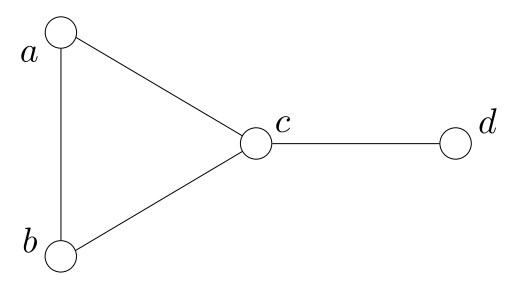

Fig. 2: A graph with four vertices

Consider the graph $G$ shown in Figure 2. The lattice $\Pi_{G}$ of forbidden colorings of $G$ and the values of the Möbius function $\mu(\hat{0}, \pi)$ for this lattice is presented in Figure 3. The resulting polynomial is

$$
P(G ; x, y)=x^{4}-4 x^{2} y+4 x y+y^{2}-2 y .
$$

In Eq. (5) the sum is extended over all blocks $\pi \in \Pi_{G}$. In the worst case this gives $B(n)$ terms, where $B(n)$ denotes the $n$th Bell number. A combinatorial interpretation of the coefficients is given in the next section. 


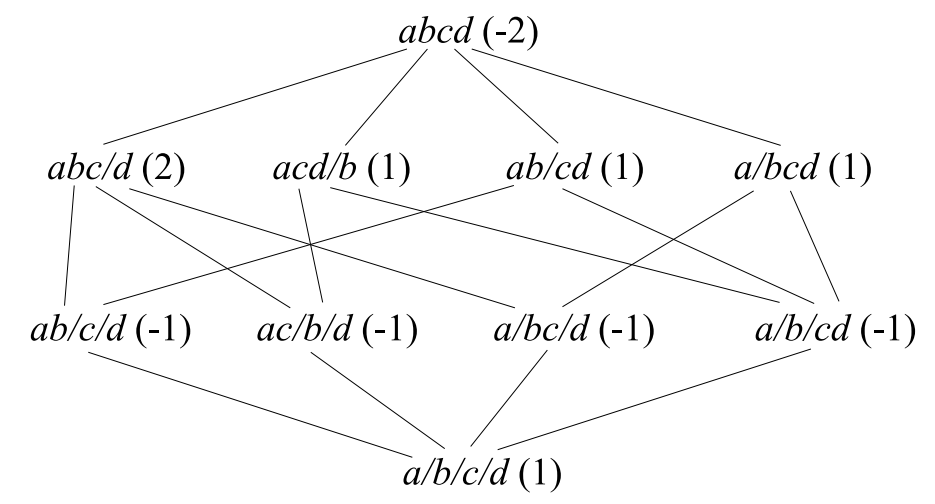

Fig. 3: Lattice of forbidden colorings for the graph in Figure \&.

STANLEY [11] defines the chromatic symmetric function for any graph $G=(V, E)$ by

$$
X_{G}:=\sum_{\kappa} \prod_{i} x_{i}^{\left|\kappa^{-1}(i)\right|}
$$

where the sum is over all proper colorings $\kappa: V \rightarrow\{1, \ldots, n\}$ of $G$. Thus, the coefficient of $x_{1}^{\lambda_{1}} \cdots x_{r}^{\lambda_{r}}$ in $X_{G}$ is the number of proper colorings of $G$ such that $\lambda_{k}$ vertices are colored $k$ for $k=1, \ldots, r$. Stanley shows that

$$
X_{G}=\sum_{\pi \in \Pi_{G}} \mu(\hat{0}, \pi) p_{\lambda(\pi)}
$$

where $\lambda(\pi)$ denotes the type of $\pi$, that is, $\lambda(\pi)=\left(\lambda_{1}, \ldots, \lambda_{r}\right)$ where the $\lambda_{k}$ 's denote the sizes of the blocks of $\pi$, and where $p$ denotes the power sum symmetric function, which for $\lambda=\left(\lambda_{1}, \ldots, \lambda_{r}\right)$ is defined by (cf. [11])

$$
p_{\lambda}:=\prod_{k=1}^{|\lambda|} \sum_{i} x_{i}^{\lambda_{k}},
$$

where $|\lambda|$ denotes the number of parts of $\lambda$. In view of this and Theorem 4 , the coefficients of our generalized chromatic polynomial and of Stanley's chromatic symmetric function are related via the lattice $\Pi_{G}$.

We proceed by establishing a connection between our polynomial and the matching polynomial. Recall that a matching of a graph $G=(V, E)$ is a subset $F$ of $E$ such that no two edges of $F$ share a common vertex. Let $m_{k}$ be the number of matchings of cardinality $k$ in $G$. The matching polynomial of $G$ is defined by

$$
M(G ; x):=\sum_{k=0}^{\lfloor n / 2\rfloor}(-1)^{k} m_{k} x^{n-2 k} .
$$

Corollary 5 The matching polynomial is related to the generalized chromatic polynomial via

$$
\left[z^{n-2 k}\right] M(G ; z)=\left[x^{n-2 k} y^{k}\right] P(G ; x, y),
$$


where $\left[z^{n-2 k}\right] M(G ; z)$ and $\left[x^{n-2 k} y^{k}\right] P(G ; x, y)$ denote the coefficients of $z^{n-2 k}$ in $M(G ; z)$ and $x^{n-2 k} y^{k}$ in $P(G ; x, y)$, respectively.

Proof. By Theorem 4 we have

$$
\left[x^{n-2 k} y^{k}\right] P(G ; x, y)=\sum_{\substack{\pi \in \Pi_{G} \\ k_{1}(\pi)=n-2 k \\|\pi|=n-k}} \mu(\hat{0}, \pi)
$$

where the Möbius function of an interval $[\hat{0}, \pi]$ in the partition lattice is given by (c.f. RoTA [8] $)$

$$
\mu(\hat{0}, \pi)=(-1)^{n-|\pi|} \prod_{A \in \pi}(|A|-1) !
$$

Here $A \in \pi$ indicates that $A$ is a block of $\pi$. A partition of $\{1, \ldots, n\}$ with exactly $n-2 k$ singletons and $n-k$ blocks must contain exactly $k$ two-element blocks. If $\pi$ is such a partition, then Eq. (7) yields

$$
\mu(\hat{0}, \pi)=(-1)^{k} .
$$

This is also the value of the Möbius function in $\Pi_{G}$ since the interval $[\hat{0}, \pi]$ coincides with the corresponding interval of the partition lattice. Thus, Eq. (6) becomes

$$
\left[x^{n-2 k} y^{k}\right] P(G ; x, y)=(-1)^{k}\left|\left\{\pi \in \Pi_{G}: k_{1}(\pi)=n-2 k,|\pi|=n-k\right\}\right| .
$$

Since there is a one-to-one correspondence between the set of partitions on the right-hand side of the formula and the set of matchings of cardinality $k$, the statement of the corollary immediately follows.

Thus the generalized chromatic polynomial $P(G ; x, y)$ incorporates the matching polynomial of $G$ as well as the independence polynomial of $G$. The matching polynomial and the independence polynomial are also related by the line graph via the identity (cf. [5] )

$$
M(G ; x)=(-1)^{n} x^{n-2 m} I\left(L(G) ;-x^{2}\right),
$$

but using $P(G ; x, y)$ eliminates the need for using $L(G)$. Thus, our new polynomial may be considered as a generalization of the chromatic polynomial, the independence polynomial, and the matching polynomial.

We proceed by establishing a representation of our new polynomial in terms of falling factorials. A partition $\pi$ of the vertex set $V(G)$ is called independent if every block of $\pi$ is an independent set of $G$. We use $\lambda \vdash n$ to denote that $\lambda$ is a number partition of $n$. We obtain the following partition representation:

Theorem 6 For each $\lambda \vdash n=|V(G)|$, let $a_{\lambda}$ be the number of independent partitions of $G$ of type $\lambda$. Then, the generalized chromatic polynomial satisfies

$$
\left.P(G ; x, y)=\sum_{\lambda \vdash n} a_{\lambda} y \frac{|\lambda|-k_{1}(\lambda)}{k_{1}(\lambda)} \sum_{k=0}^{k_{1}(\lambda)} \begin{array}{c} 
\\
k
\end{array}\right)(x-y)^{k}\left(y-|\lambda|+k_{1}(\lambda)\right) \frac{k_{1}(\lambda)-k}{},
$$

where $y^{i}$ denotes the falling factorial $y(y-1) \cdots(y-i+1)$. 
Proof. The formula counts all proper generalized colorings of $G$. To avoid multiple counting, we color all vertices of each block having more than one vertex by one proper color of $\mathcal{Y}$. Different blocks


blocks. The remaining singletons of the partition may be colored arbitrarily with proper colors or improper ones. When using proper colors the singletons must be colored differently. This gives the second sum on the right-hand side, and we are done.

Corollary 7 Let $a_{i j}$ be the number of independent partitions of $G$ with exactly $i$ singletons and $j$ blocks having two or more vertices. Then, the following equation holds:

$$
P(G ; x, y)=\sum_{i=0}^{n} \sum_{j=0}^{n} a_{i j} \sum_{k=0}^{i}\left(\begin{array}{l}
i \\
k
\end{array}\right)(x-y)^{k} y^{i+j-k} .
$$

Corollary 7 suggests the introduction of a simpler two-variable polynomial of the form

$$
Q(G ; x, y)=\sum_{i=0}^{n} \sum_{j=0}^{n} a_{i j} x^{i} y^{j},
$$

which comprises the same amount of information as our generalized chromatic polynomial. However, the simplicity of defining the polynomial in this way has to be paid for with loss of many nice properties, e.g., the multiplicity with respect to components.

\section{The coefficients in terms of broken circuits}

One of the most important results about the usual chromatic polynomial is Whitney's broken circuit theorem [15], which expresses the coefficients of the chromatic polynomial in terms of broken circuits. Many results can be deduced directly from Whitney's broken circuit theorem; see e.g., LAZEBNIK [6].

Let $G=(V, E)$ be a graph where $|V|=n$ and $E$ is linearly ordered. A broken circuit of $G$ is obtained from the edge set of a cycle of $G$ by removing its maximum edge. The broken circuit complex of $G$, abbreviated to $\mathcal{B C}(G)$, is the set of all non-empty subsets of the edge set not including any broken circuit as a subset. The definition of a broken circuit goes back to WHITNEY [15], while the broken circuit complex was initiated by WILF [16] and further investigated by BRYLAWSKI and OXLEY [2, 3]. In fact, $\mathcal{B C}(G)$ is an abstract simplicial complex in the sense of topology, whence we refer to each $I \in \mathcal{B} C(G)$ as a face of $\mathcal{B C}(G)$.

Whitney's broken circuit theorem [15] states that for any $y \in \mathbb{N}$,

$$
P(G ; y)=\sum_{k=0}^{n}(-1)^{k} b_{k} y^{n-k}
$$

where $b_{0}=1$ and $b_{k}, k>0$, counts the faces of cardinality $k$ in the broken circuit complex of $G$. Note that while the definition of the broken circuit complex depends on the ordering of the edges, the same does not apply to the $b_{k}$ 's.

We now generalize Whitney's broken circuit theorem to our new two-variable polynomial $P(G ; x, y)$. The proof of this generalization (and thus of Whitney's original result) is facilitated by applying the following inclusion-exclusion variant, which is an immediate consequence of [4, Corollary 3.5]. 
Proposition 8 Let $\left\{A_{e}\right\}_{e \in E}$ be a finite family of finite sets, where $E$ is endowed with a linear ordering relation. Furthermore, let $\mathcal{F}$ be a set of non-empty subsets of $E$ such that for any $F \in \mathcal{F}$,

$$
\bigcap_{i \in F} A_{i} \subseteq \bigcup_{j>\max F} A_{j}
$$

Then,

$$
\left|\bigcup_{e \in E} A_{e}\right|=\sum_{\substack{I \subseteq E, I \neq \emptyset \\ I \not \geq F}(\forall F \in \mathcal{F})}(-1)^{|I|-1}\left|\bigcap_{i \in I} A_{i}\right| .
$$

For any graph $G=(V, E)$ and any subset $I$ of $E$ we use $G[I]$ to denote the graph having vertex set $\{v \in V \mid v \in e$ for some $e \in I\}$ and edge set $I$.

We are now ready to state the main result of this section, which coincides with Whitney's broken circuit theorem in the diagonal case where $x=y$.

Theorem 9 Let $G=(V, E)$ be a graph with $n$ vertices and $m$ edges and whose edge set is endowed with a linear ordering relation. Furthermore, let $x \in \mathbb{N}$ and $y \in\{0, \ldots, x\}$. Then,

$$
P(G ; x, y)=\sum_{k=0}^{m} \sum_{l=0}^{k}(-1)^{k} b_{k l} x^{n-k-l} y^{l},
$$

where $b_{00}=1$ and $b_{k l}, k>0$, counts all faces I of cardinality $k$ in the broken circuit complex of $G$ such that $G[I]$ has exactly $l$ connected components.

Proof. Define $\mathcal{F}$ as the set of broken circuits of $G$ and for any edge $e$ of $G$ define

$$
A_{e}:=\{f: V \rightarrow\{1, \ldots, x\} \mid f(u)=f(v) \leq y\}, \quad e=\{u, v\} .
$$

Then, the requirements of Proposition $\$$ are satisfied, and thus we obtain

$$
P(G ; x, y)=x^{n}-\left|\bigcup_{e \in E} A_{e}\right|=x^{n}+\sum_{k=1}^{m}(-1)^{k} \sum_{\substack{I \in \mathcal{B} C(G) \\|I|=k}}\left|\bigcap_{i \in I} A_{i}\right| .
$$

For any subset $I \in \mathcal{B C}(G)$ let $m_{I}, n_{I}$ and $c_{I}$ denote the number of edges, vertices and connected components of the edge-subgraph $G[I]$, respectively. Since $G[I]$ is cycle-free for any $I \in \mathcal{B C}(G)$, we conclude that $m_{I}-n_{I}+c_{I}=0$ and hence,

$$
\left|\bigcap_{i \in I} A_{i}\right|=x^{n-n_{I}} y^{c_{I}}=x^{n-m_{I}-c_{I}} y^{c_{I}} .
$$

Now, put (11) into (10) and take into account that $c_{I} \leq m_{I}$ and $m_{I}=|I|$.

In view of the results in [4] it can even be shown that for any $r \in \mathbb{N}$,

$$
\begin{aligned}
& P(G ; x, y) \leq \sum_{k=0}^{r} \sum_{l=0}^{k}(-1)^{k} b_{k l} x^{n-k-l} y^{l} \quad(r \text { odd }) \\
& P(G ; x, y) \geq \sum_{k=0}^{r} \sum_{l=0}^{k}(-1)^{k} b_{k l} x^{n-k-l} y^{l} \quad(r \text { even })
\end{aligned}
$$


where $b_{k l}$ is defined as in Theorem 9. The proof of this 'Bonferroni-like' result is left to the reader.

We further remark that the independence polynomial $I(G ; x)$ satisfies

$$
I(G ; x)=\sum_{k=0}^{m} \sum_{l=0}^{k}(-1)^{k} b_{k l}(x+1)^{n-k-l},
$$

which immediately follows from $I(G ; x)=P(G ; x+1,1)$ and Theorem 9, and that (9) can be restated as

$$
P(G ; x, y)=x^{n} Z\left(G ; \frac{1}{x}, \frac{y}{x}\right),
$$

where

$$
Z(G ; x, y):=\sum_{k=0}^{m} \sum_{l=0}^{k}(-1)^{k} b_{k l} x^{k} y^{l} .
$$

Thus, $y^{n} Z\left(G ; y^{-1}, 1\right)$ and $(x+1)^{n} Z\left(G ;(x+1)^{-1},(x+1)^{-1}\right)$ give the chromatic polynomial and the independence polynomial of $G$, respectively.

As an example, consider the path $P_{4}$ and the star $S_{3}$ in Figure 1. Let $b_{k l}$ and $b_{k l}^{\prime}$ denote the coefficients of $P\left(P_{4} ; x, y\right)$ and $P\left(S_{3} ; x, y\right)$, respectively. According to the interpretation of the coefficients provided by Theorem $Q$ we find that

$$
\begin{aligned}
& b_{00}=1, \quad b_{22}=1, \quad b_{00}^{\prime}=1, \quad b_{22}^{\prime}=0 \text {, } \\
& b_{10}=0, \quad b_{30}=0, \quad b_{10}^{\prime}=0, \quad b_{30}^{\prime}=0 \text {, } \\
& b_{11}=3, \quad b_{31}=1, \quad b_{11}^{\prime}=3, \quad b_{31}^{\prime}=1 \text {, } \\
& b_{20}=0, \quad b_{32}=0, \quad b_{20}^{\prime}=0, \quad b_{32}^{\prime}=0 \text {, } \\
& b_{21}=2, \quad b_{33}=0, \quad b_{21}^{\prime}=3, \quad b_{33}^{\prime}=0 \text {. }
\end{aligned}
$$

Putting these values into (9) we again obtain (3) and (4). The corresponding $Z$-polynomials are

$$
\begin{aligned}
& Z\left(P_{4} ; x, y\right)=1-3 x y+2 x^{2} y+x^{2} y^{2}-x^{3} y \\
& Z\left(S_{3} ; x, y\right)=1-3 x y+3 x^{2} y-x^{3} y .
\end{aligned}
$$

\section{Special graphs}

\subsection{The complete graph}

The lattice $\Pi_{G}$ for the complete graph $G=K_{n}$ coincides with the partition lattice $\Pi(V)$ of $V$, where $V$ denotes the vertex set of $G$. From (5) we obtain the equation

$$
P\left(K_{n} ; x, y\right)=\sum_{\pi \in \Pi(V)} \mu(\hat{0}, \pi) x^{k_{1}(\pi)} y^{|\pi|-k_{1}(\pi)},
$$

where $\mu$ is the Möbius function of the partition lattice as defined in Eq. (7). A closer look at Eq. (12) reveals that the terms of the sum do not depend on the partition $\pi$ but only on its type. In the following, we use a 
second representation for a number partition $\lambda$. Besides $\lambda=\left(\lambda_{1}, \ldots, \lambda_{r}\right)$ we also write $\lambda=\left(1^{k_{1}} 2^{k_{2}} \ldots n^{k_{n}}\right)$ where each $k_{i}$ gives the number of parts of size $i$ in $\lambda$. Consequently,

$$
\sum_{i=1}^{n} k_{i}=|\lambda| \text { and } \sum_{i=1}^{n} i k_{i}=n
$$

and hence,

$$
P\left(K_{n} ; x, y\right)=n ! \sum_{\lambda \vdash n} \frac{(-1)^{n-|\lambda|}}{\prod_{i=1}^{|\lambda|} \lambda_{i} \prod_{i=1}^{n} k_{i} !} x^{k_{1}} y^{|\lambda|-k_{1}} .
$$

A simpler representation of this formula follows immediately from Corollary ฤ:

$$
P\left(K_{n} ; x, y\right)=\sum_{k=0}^{n}\left(\begin{array}{l}
n \\
k
\end{array}\right)(x-y)^{k} y^{n-k} .
$$

\subsection{The complete bipartite graph}

Let $K_{m, n}=(V \cup W, E)$ be the complete bipartite graph with $m+n=|V|+|W|$ vertices. We choose $k$ vertices from $V$ and color these vertices with proper colors. Let $\left\{\begin{array}{l}k \\ j\end{array}\right\}$ denote the Stirling number of the second kind. For $j$ different colors there are $\left\{\begin{array}{c}k \\ j\end{array}\right\} y^{j}$ different colorings. The remaining $m-k$ vertices of $V$ may be colored with improper colors. No vertex of $W$ may receive one of the $j$ proper colors that are used for $V$. Hence, we count $(x-j)^{n}$ different colorings for vertices of $W$. Summing up all possibilities, we obtain

$$
P\left(K_{m, n} ; x, y\right)=\sum_{k=0}^{m}\left(\begin{array}{c}
m \\
k
\end{array}\right)(x-y)^{m-k} \sum_{j=0}^{k}\left\{\begin{array}{l}
k \\
j
\end{array}\right\} y^{\underline{j}}(x-j)^{n} .
$$

As a special case, we obtain for $S_{n}=K_{1, n}$ :

$$
P\left(S_{n} ; x, y\right)=x^{n}(x-y)+y(x-1)^{n} .
$$

\subsection{The path}

Let $P_{n}$ be the path with $n$ vertices. As an example, we first consider the path $P_{5}$ as shown in Figure 4 .

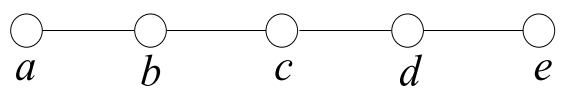

Fig. 4: The path $P_{5}$

The lattice of forbidden colorings for $P_{5}$ is shown in Figure 5. We observe that this lattice is isomorphic to the Boolean lattice $\mathbf{B}_{4}$. More generally, the lattice $\Pi_{P_{n}}$ is isomorphic to the Boolean lattice $\mathbf{B}_{n-1}$ (in fact, $\Pi_{T}$ is isomorphic to $\mathbf{B}_{n-1}$ for any tree $T$ having $n$ vertices). The proof of this fact is rather easy. A connected partition of the vertex set of the path $P_{n}$ with $k$ blocks is generated by choosing $k-1$ separators 


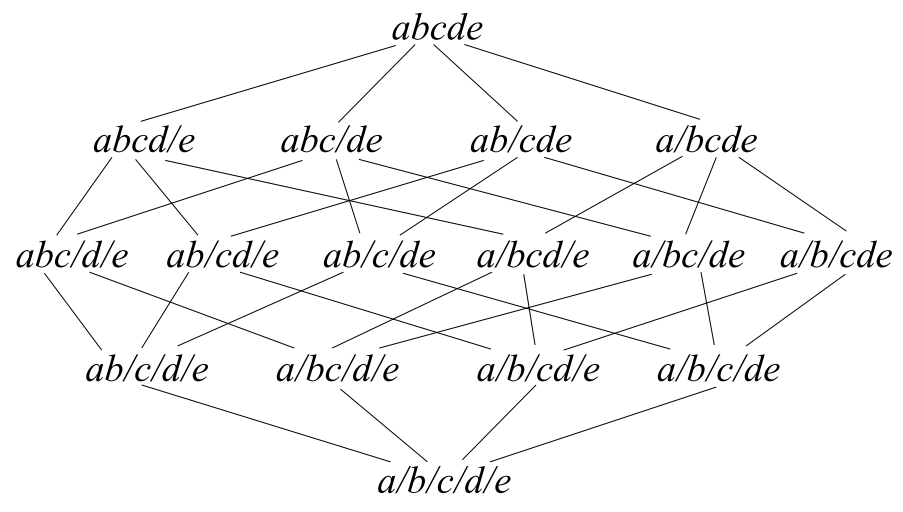

Fig. 5: The lattice of forbidden colorings for $P_{5}$

between the elements of the linearly ordered set $\left\{v_{1}, \ldots, v_{n}\right\}$. A separator corresponds to a removed edge of the path. In the following, we refer to such a partition as a linear partition. Now, by Theorem $\square$,

$$
P\left(P_{n} ; x, y\right)=\sum_{i, j}(-1)^{n-i-j} l_{i j} x^{i} y^{j}
$$

where $l_{i j}$ denotes the number of linear partitions $\pi$ of $v_{1}, v_{2}, \ldots, v_{n}$ with $i$ singletons blocks and $j$ nonsingleton blocks. All such $\pi$ can be constructed as follows. Start with a diagram of $i+j-1$ slashes which create $i+j$ spaces (between, before and after slashes) in which to place dots. Place one dot in $i$ of the spaces and two dots in the remaining $j$. This can be done in $\left(\begin{array}{c}i+j \\ i\end{array}\right)$ ways. Now distribute $n-i-2 j$ dots among the spaces that already have two dots. The number of ways of doing this is the number of ways of choosing $n-i-2 j$ objects from $j$ objects with repetition which equals

$$
\left(\begin{array}{c}
j+(n-i-2 j)-1 \\
n-i-2 j
\end{array}\right)=\left(\begin{array}{c}
n-i-j-1 \\
n-i-2 j
\end{array}\right) .
$$

Now replace the $n$ dots from left to right with the labels $v_{1}, v_{2}, \ldots, v_{n}$. The result is a linear partition with the specified number of singleton and non-singleton blocks and every such partition is obtainable in this way. Note that the total number of choices is

$$
l_{i j}=\left(\begin{array}{c}
i+j \\
i
\end{array}\right)\left(\begin{array}{c}
n-i-j-1 \\
n-i-2 j
\end{array}\right)
$$

as desired. Hence,

$$
P\left(P_{n} ; x, y\right)=\sum_{0<i+2 j \leq n}(-1)^{n-i-j}\left(\begin{array}{c}
i+j \\
i
\end{array}\right)\left(\begin{array}{c}
n-i-j-1 \\
n-i-2 j
\end{array}\right) x^{i} y^{j}
$$


For example, the polynomials of paths of up to five vertices are

$$
\begin{aligned}
& P\left(P_{1} ; x, y\right)=x \\
& P\left(P_{2} ; x, y\right)=x^{2}-y \\
& P\left(P_{3} ; x, y\right)=x^{3}-2 x y+y \\
& P\left(P_{4} ; x, y\right)=x^{4}-3 x^{2} y+y^{2}+2 x y-y \\
& P\left(P_{5} ; x, y\right)=x^{5}-4 x^{3} y+3 x y^{2}+3 x^{2} y-2 y^{2}-2 x y+y
\end{aligned}
$$

Section 7 gives a method for obtaining the generalized chromatic polynomial of any tree by using a polynomial-time recursive algorithm.

\subsection{The cycle}

The last special graph to be considered here is the cycle $C_{n}$. The construction of the lattice of forbidden colorings differs only slightly from the construction of the corresponding lattice for the path. Let $V=$ $\left\{v_{1}, \ldots, v_{n}\right\}$ be the vertex set of $C_{n}$ in correspondence with the order of traversing the cycle. A cyclic partition $\pi$ of $V$ is one obtained by removing edges from $C_{n}$ and using the resulting connected subsets of vertices as blocks of $\pi$. Note that the lattice of cyclic partitions of $V$ is isomorphic to $\mathbf{B}_{n}$ with the rank just below the maximum element removed. By Theorem 4 ,

$$
P\left(C_{n} ; x, y\right)=(n-1)(-1)^{n-1} y+\sum_{(i, j) \neq(0,1)}(-1)^{n-i-j} c_{i j} x^{i} y^{j}
$$

where $c_{i j}$ denotes the number of cyclic partitions which have $i$ singletons and $j$ non-singletons. These can be obtained as follows. As in Section 5.3, build a dot diagram which can be done in $l_{i j}$ ways where $l_{i j}$ again denotes the number of linear partitions. Now turn the diagram into a cyclic partition by picking one of the $n$ dots and then replacing the dots starting with that dot as $v_{1}$, moving right, and then wrapping around circularly to get the dots to the left of $v_{1}$. Thus we get $n l_{i j}$ labeled diagrams. We claim that every cyclic partition is obtained exactly $i+j$ times. This is because any circular partition can be written with the element $v_{1}$ in any of the $i+j$ blocks. So $n l_{i j}=(i+j) c_{i j}$ and hence,

$$
c_{i j}=\frac{n}{i+j}\left(\begin{array}{c}
i+j \\
i
\end{array}\right)\left(\begin{array}{c}
n-i-j-1 \\
n-i-2 j
\end{array}\right) .
$$

Therefore,

$$
P\left(C_{n} ; x, y\right)=(-1)^{n} y+n \sum_{0<i+2 j \leq n} \frac{(-1)^{n-i-j}}{i+j}\left(\begin{array}{c}
i+j \\
i
\end{array}\right)\left(\begin{array}{c}
n-i-j-1 \\
n-i-2 j
\end{array}\right) x^{i} y^{j} .
$$

For example, the generalized chromatic polynomial for cycles of up to five vertices are

$$
\begin{aligned}
& P\left(C_{3} ; x, y\right)=x^{3}-3 x y+2 y, \\
& P\left(C_{4} ; x, y\right)=x^{4}-4 x^{2} y+4 x y+2 y^{2}-3 y, \\
& P\left(C_{5} ; x, y\right)=x^{5}-5 x^{3} y+5 x y^{2}+5 x^{2} y-5 y^{2}-5 x y+4 y .
\end{aligned}
$$




\section{Non-isomorphic graphs}

Does the generalized chromatic polynomial distinguish non-isomorphic graphs? Two non-isomorphic graphs having the same generalized chromatic polynomial must have the same chromatic polynomial, the same matching polynomial, and the same independence polynomial. Consequently, the number of vertices, edges, and components must be the same for each such pair. The smallest non-isomorphic graphs having the same generalized chromatic polynomial are depicted in Figure 6. We found by complete
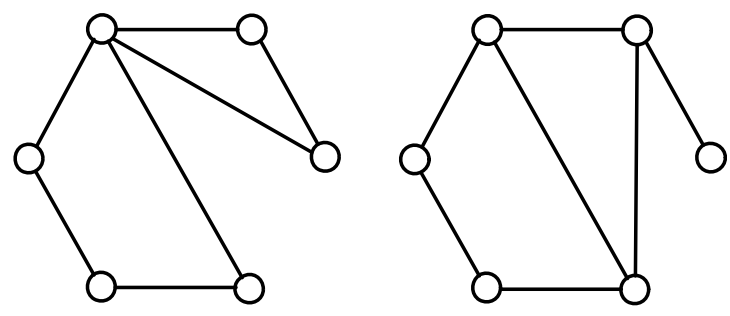

Fig. 6: Non-isomorphic graphs having the same generalized chromatic polynomial.

enumeration that there is no pair of non-isomorphic trees with up to nine vertices that have the same generalized chromatic polynomial. We found such a pair with 10 vertices which is presented in Figure 7 . Note that the chromatic symmetric function, as defined by STANLEY [11], does not coincide for these two

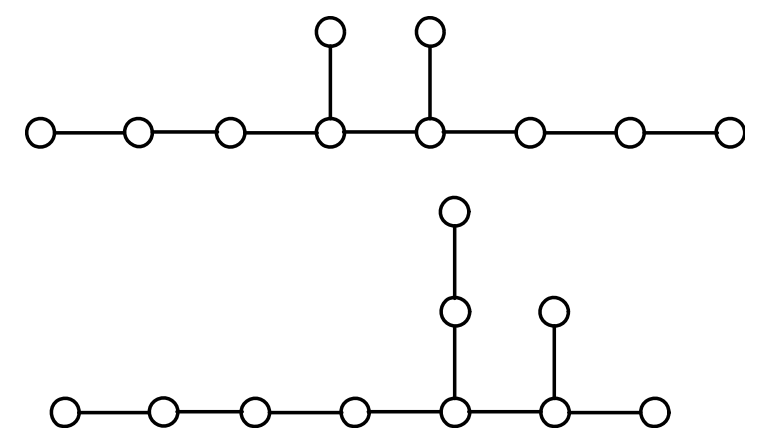

Fig. 7: Non-isomorphic trees having the same generalized chromatic polynomial.

trees. The question whether the chromatic symmetric function distinguishes non-isomorphic trees is still open. We hope that our polynomial could be of some value to decide this question. The search for pairs of non-isomorphic trees having the same chromatic symmetric function can now be restricted to those pairs of trees that have the same generalized chromatic polynomial. In this way (and using a computer) we found that there are no non-isomorphic trees with up to 15 vertices that have the same chromatic symmetric function. 


\section{A polynomial algorithm for trees}

As with the usual chromatic polynomial, the computation of the generalized chromatic polynomial is an NP-hard (in fact: \#P-complete) counting problem. As a consequence, polynomial-time algorithms can only be found for some restricted classes of graphs. In this section, we consider the restricted class of trees.

Let $P(G, W ; x, y)$ be the generalized chromatic polynomial of $G$ with the additional restriction that there is one proper color forbidden for all vertices of some given subset $W$ of $V$. Let $C(G, v)$ be the set of components of $G-v$. The following theorem provides an algorithm for computing $P(G ; x, y)$ for any tree $G$.

Theorem 10 Let $T$ be a tree and $v \in V(T)$. Then, for each $T_{i} \in C(G, v)$ the set $N(v) \cap V\left(T_{i}\right)$ consists of only one vertex, and the polynomial $P(T ; x, y)$ can be computed via the following recursion:

$$
\begin{gathered}
P(T ; x, y)=(x-y) \prod_{T_{i} \in C(T, v)} P\left(T_{i} ; x, y\right)+y \prod_{T_{i} \in C(T, v)} P\left(T_{i}, N(v) \cap V\left(T_{i}\right) ; x, y\right), \\
P(T,\{v\} ; x, y)=(x-y) \prod_{T_{i} \in C(T, v)} P\left(T_{i} ; x, y\right)+(y-1) \prod_{T_{i} \in C(T, v)} P\left(T_{i}, N(v) \cap V\left(T_{i}\right) ; x, y\right) .
\end{gathered}
$$

If $T$ only consists of $v$, then

$$
\begin{gathered}
P(T ; x, y)=x, \\
P(T,\{v\} ; x, y)=x-1 .
\end{gathered}
$$

Proof. Consider the first equation. Each vertex of $V$ can be colored with proper or improper colors. There are exactly $x-y$ possibilities to choose an improper color for $v$ in which case the coloring of the neighbors of $v$ is independent of the color of $v$. Consequently, $v$ can be removed from $G$. The number of remaining colorings follows from the product formula (2). The second product of the first equation arises from the $y$ different colorings of $v$ with proper colors. Now, the neighbors of $v$ have to be colored differently from $v$. The neighbor of $v$ in the subtree $T_{i}$ is given by $N(v) \cap V\left(T_{i}\right)$. Indeed, this set contains exactly one vertex. The number of remaining colorings of the subtree $T_{i}$ are given by $P\left(T_{i}, N(v) \cap V\left(T_{i}\right) ; x, y\right)$. This shows the first formula. In order to prove the second formula we first remark that there are again $x-y$ different colorings using only improper colors, which cause no restriction on the components of $T-v$. However, if we color $v$ with a proper color, then we have only $y-1$ possibilities. The remaining part is proved in a similar way.

Figure 8 illustrates the computation of the generalized chromatic polynomial for an example tree. The darkened vertices in this figure correspond to the vertices for which one color is forbidden. The decomposition vertex at each step is denoted by $v$. The resulting polynomial for the depicted tree is:

$$
\begin{aligned}
P(T ; x, y) & =(x-y) x^{3}\left[(x-y) x^{2}+y(x-1)^{2}\right]+y(x-1)^{3}\left[(x-y) x^{2}+(y-1)(x-1)^{2}\right] \\
& =x^{7}-6 x^{5} y+9 x^{4} y+6 x^{3} y^{2}-11 x^{3} y-9 x^{2} y^{2}+10 x^{2} y+5 x y^{2}-5 x y-y^{2}+y .
\end{aligned}
$$

There exists a vertex $v$ in every tree with $n$ vertices such that no component of $T-v$ has more than $n / 2$ vertices. This vertex can be found in polynomial time as follows. Start with a randomly chosen decomposition vertex $w$. If $T-w$ contains a component $T_{i}$ with more the $n / 2$ vertices, then next choose 


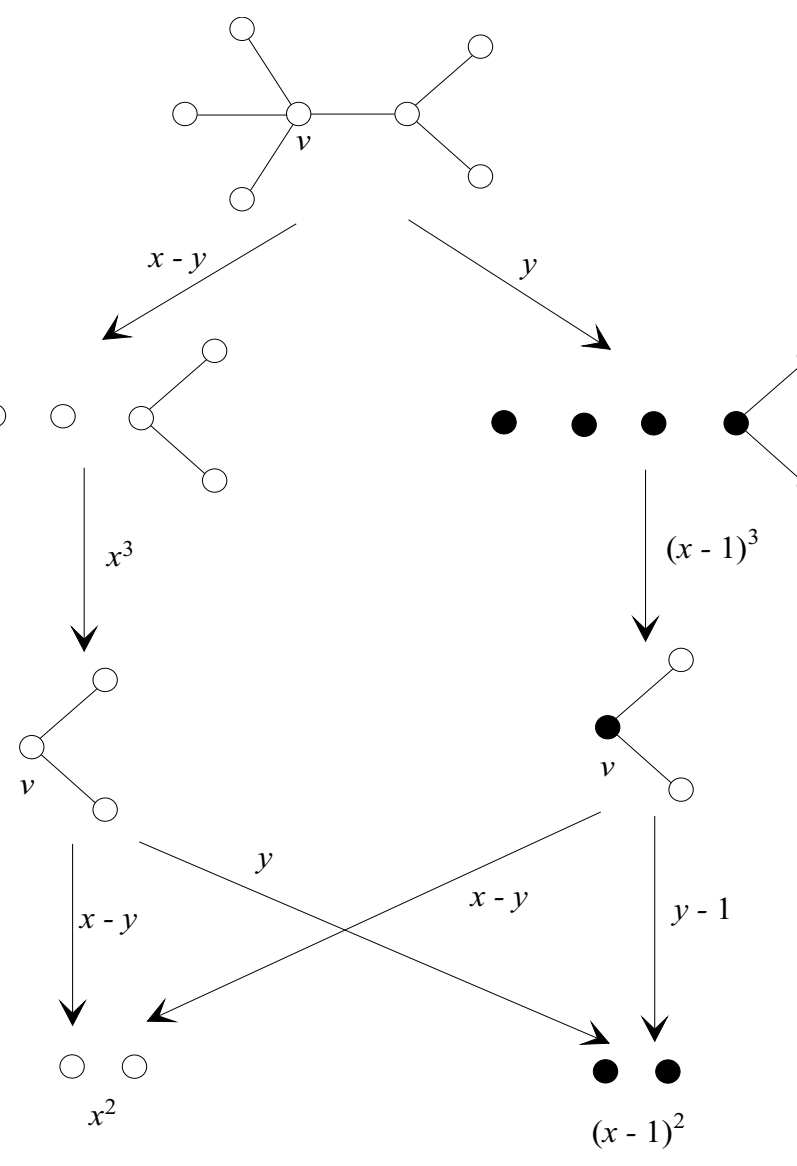

Fig. 8: Computation of the generalized chromatic polynomial of a tree

the neighbor of $w$ belonging to $T_{i}$. Repeating this procedure, we obtain after at most $n / 2$ steps a maximum component with at most $n / 2$ vertices. By the appropriate combination of components, we can always achieve a decomposition into three subtrees each consisting of at most $n / 2$ vertices.

Let $f(n)$ be the time complexity of the recursive algorithm as described in Theorem 10 for the computation of the generalized chromatic polynomial of any input tree of size $n=|V|$. We find that

$$
f(n) \leq 3 f\left(\frac{n}{2}\right)+p(n)
$$

where $p(n)$ is a polynomial that incorporates the effort of the search for a decomposition vertex. For each polynomial $p(n)$ inequality (13) leads to solutions which are polynomial in $n$. Consequently, we obtain a polynomial time algorithm for the computation of the generalized chromatic polynomial of a tree:

Corollary 11 The generalized chromatic polynomial of a tree can be computed in polynomial time. 
Corollary 12 The generalized chromatic polynomial of a path $P_{n}, n>1$, satisfies the recurrence relation

$$
P\left(P_{n} ; x, y\right)=(x-y) P\left(P_{n-1} ; x, y\right)+y(y-1)^{n-2}(x-1)+y(x-y) \sum_{i=2}^{n-1}(y-1)^{i-2} P\left(P_{n-i} ; x, y\right)
$$

with the initial condition $P\left(P_{1} ; x, y\right)=x$.

Corollary 12 is an immediate consequence of Theorem 10 .

\section{Graphs of restricted pathwidth}

Many NP-hard graph problems become simple in graphs of restricted treewidth or pathwidth. A nice introduction to treewidth and related problems is given by BODLAENDER [1]. Here we present a polynomialtime algorithm for the computation of the generalized chromatic polynomial for graphs of restricted pathwidth. The main ideas may also be extended to cover graphs of restricted treewidth. The problem of determining the pathwidth (treewidth) is NP-hard in general. If the pathwidth is known to be at most $k$ then there is a polynomial-time algorithm for finding a path decomposition (composition order) of width $k$.

A path decomposition of a graph $G=(V, E)$ is a sequence $\mathcal{I}=\left(J_{0}, \ldots, J_{r}\right)$ of subsets of $V$ such that

1. $\bigcup_{i=0}^{r} J_{i}=V, J_{0}=J_{r}=\emptyset$,

2. every edge of $G$ has both its vertices contained in at least one subset of $\mathcal{I}$,

3. for all $i, j, k$ with $0 \leq i<j<k \leq r$ the relation $J_{i} \cap J_{k} \subseteq J_{j}$ holds.

The width of a path decomposition $\mathcal{I}$ is the maximal cardinality of a subset of $\mathcal{I}$. The pathwidth of the graph $G$ is the minimum width over all path decompositions of $G$. A path decomposition $\mathcal{I}=\left(J_{0}, \ldots, J_{r}\right)$ is called nice if $\left|\left(J_{i+1} \backslash J_{i}\right) \cup\left(J_{i} \backslash J_{i+1}\right)\right|=1$ for all $i=0, \ldots, r-1$. A nice path decomposition $\mathcal{J}$ may be described in terms of a sequence $S(g)$ of signed vertices of $G$ : The notation $+v$ indicates the inclusion of the vertex $v$ into the set $J_{i}$ while the notation $-v$ stands for the removal of $v$ from $J_{i}$.

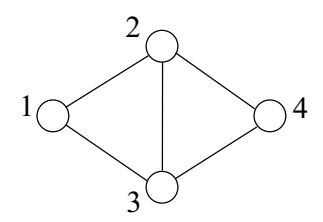

Fig. 9: Bridge graph.

For example, a nice path decomposition of the bridge graph presented in Figure 9 is

$$
\mathcal{I}=(\emptyset,\{1\},\{1,2\},\{1,2,3\},\{2,3\},\{2,3,4\},\{3,4\},\{4\}, \emptyset) .
$$

which is uniquely determined by the following sequence of signed vertices

$$
S(\mathcal{g})=(+1,+2,+3,-1,+4,-2,-3,-4)
$$


It follows from the definition of path decomposition that each vertex appears exactly once with positive sign and exactly once with negative sign in $S(\mathcal{J})$. Let $\mathcal{J}=\left(J_{0}, \ldots, J_{r}\right)$ a path decomposition of width $k$ of $G$. Then $\mathcal{I}$ can be transformed in $O(n)$ time into a nice path decomposition $\tilde{\mathcal{J}}$ of width at most $k$ (see [9] and [12]).

A composition order of a graph $G=(V, E)$ is a sequence $s=\left(s_{1}, \ldots, s_{t}\right)$ of signed vertices and of edges of $G$ such that

1. if $s_{i} \in s$ represents an edge with end vertices $u$ and $v$ then there are indices $j, k, m, p$ with $j, k<i<$ $m, p$ and $s_{j}=u, s_{k}=v, s_{m}=-u, s_{p}=-v$,

2. every edge of $G$ appears exactly once in $s$,

3. the removal of all edges from $s$ yields a nice path decomposition of $G$.

A composition order of the graph in Figure 9 is

$$
s=(+1,+2,\{1,2\},+3,\{1,3\},-1,\{2,3\},+4,\{2,4\},-2,\{3,4\},-3,-4) .
$$

We refer to the objects in a composition order as steps or, to be more precise, as vertex activation steps (positively signed vertices), vertex deactivation steps (negatively signed vertices), and edge insertion steps. The composition order gives complete information about the graph structure, that is, no other graph representation is required for the computation of the generalized chromatic polynomial.

Corollary 7 shows that the numbers $a_{p q}$ of independent partitions consisting of $p$ singletons and $q$ other blocks give all the information which is necessary to determine the generalized chromatic polynomial of $G$. The algorithm presented below computes these numbers directly. Some additional notation is needed. Let $G_{i}$ be the graph consisting of all activated vertices and all edges inserted up to and including step $i$ in a composition order $s$, let $V_{i}$ be the vertex set of $G_{i}$, and $A_{i}$ be the set of active vertices of $G_{i}$, i.e., $V_{i}$ minus the set of vertices deactivated up to and including step $i$. A labeled partition $\pi$ of $A_{i}$ is a set partition of $A_{i}$ where blocks may have a label that will be denoted by a star $\left(^{*}\right)$.

For each independent partition $\sigma$ of $V_{i}$ we define the induced index of $\sigma$ as a triple $I=(\pi, p, q)$ consisting of a labeled partition $\pi$ of $A_{i}$ and two integers $p$ and $q$ where $p$ equals the number of singletons of $\sigma$ and $q$ is the number of blocks of $\sigma$ with more than one vertex. The blocks of $\pi$ correspond to the restriction of $\sigma$ to $A_{i}$, and a block of $\pi$ is labeled iff the corresponding block of $\sigma$ is not a singleton. We note that different partitions of $V_{i}$ may have the same induced index and refer to these partitions as represented by the index. Let $c$ be the number of partitions of $V_{i}$ represented by the induced index $I$. A pair $(I, c)$ will be called a state consisting of the index $I$ and the value $c$, and the set of all states at step $i$ defines the state set $Z_{i}$.

The main idea behind the algorithm is to compute the state set $Z_{i}$ from the previous state set $Z_{i-1}$ according to certain rules depending on the step $s_{i}$ of the given composition order.

Obviously, the very first state set $Z_{0}$ consists only of a single state $((\emptyset, 0,0), 1)$, since $V_{0}=\emptyset$ and since there is only one partition of an empty set, and this partition contains neither singleton nor non-singleton blocks. More interestingly, the very last state set $Z_{r}$ contains all the numbers $a_{p q}$. The set of active vertices will be empty since each vertex has been activated and deactivated once. The partitions in the induced indices are partitions of this active set and hence empty partitions. The values $c$ corresponding to indices $I=(\pi, p, q)$ gives the number $a_{p q}$ of partitions of the full vertex set (since $V_{2|V|+|E|}=V$ ) with $p$ singleton and $q$ non-singleton blocks. 
The transformation rules for the state sets are as follows: For each step $s_{i}$, all states of the state set $Z_{i-1}$ are considered in turn leading to some (possibly empty) set of intermediate successor states. These states represent the same set of partitions as the original state but use the new active vertex set for their description. After the individual states are transformed, there might be sets of such intermediate successor states sharing the same index. These sets are replaced by single states using the common index and a value equal to the sum of the values of states in the set. The states remaining after this clean-up form the next state set $Z_{i}$.

Vertex activation $(+v)$ : After activation step $s_{i}$ activating some vertex $v$, this vertex $v$ is a singleton in $G_{i}$, since no edge incident to $v$ could have been introduced before $v$ became active. So $v$ is independent of all vertices in $G_{i-1}$ and hence, it can extend any independent set of $G_{i-1}$. Suppose we need to transform a state $z=(I, c)$ with $I=(\pi, p, q)$. Let $S=\left\{\sigma_{1}, \sigma_{2}, \ldots\right\}$ be the set of independent partitions consisting of $p$ singletons and $q$ non-singleton blocks that induce $I$. We then proceed as follows:

- First of all, note that the new vertex $v$ can form a singleton block of its own in any of the $\sigma_{k}$. In this case, the number of singletons increases by one, and $v$ forms a new unlabeled block of $\pi$. Everything else remains unchanged, leading to a single intermediate successor state of $((\pi \mid v, p+1, q), c)$, where $\pi \mid v$ is the partition obtained by augmenting $\pi$ by a single block $\{v\}$.

- Secondly, since $v$ is independent of all vertices in $G_{i-1}$, it is certainly independent of the vertices that constitute any of the $q$ non-singleton blocks of some $\sigma_{k}$. Hence, $v$ can be added to any such block, which results in a block of cardinality larger than one. Let $X$ be such a non-singleton block and define $Y:=X \cap A_{i-1}$. We have to distinguish two cases: (a) If $Y=\emptyset$, then $v$ will be a singleton in $(X+\{v\}) \cap A_{i}$. Since $|X \cup\{v\}|>1$ it must be marked, and thus the single successor state is $\left(\left(\pi \mid v^{*}, p, q\right), c\right)$. (b) If $Y \neq \emptyset$, then we already have parts of $X$ in the active vertex set, namely the vertices of $Y$. In this case, the successor state is $\left(\left(\pi-Y \mid(Y \cup\{v\})^{*}, p, q\right), c\right)$.

- Similarly to the above, the new vertex $v$ can be added to all $p$ singleton blocks. This works in a similar way with one exception: the singleton grows to a non-singleton block, whence we have to decrement $p$ and increment $q$ by one in the successor state.

Vertex deactivation $(-v)$ : If $I=(\pi, p, q)$ describes a set of independent partitions of $G_{i-1}$, then $(\pi-$ $v, p, q)$ describes the same set for $G_{i}$ where $\pi-v$ is $\pi-\{v\}$ in case that $v$ is a singleton resp. $\pi-X \mid X \backslash\{v\}$ in case that $v \in X$ for a larger block $X$ of $\pi$. So for deactivation, every state has a single intermediate successor state, which is obtained by removing the vertex for the partition in the index partition.

Edge insertion $\{u, v\}$ : Let $I, w$ and $\pi$ be defined as in the vertex activation step above. If $\pi$ is a partition containing a block $X$ with $\{u, v\} \subseteq X$ then this block forms no independent set in $G_{i}$ as $G_{i}$ results from $G_{i-1}$ by inserting edge $\{u, v\}$. Consequently, the state $(I, c)$ has no successor states as there are no independent partitions inducing $\pi$ in $G_{i}$.

As an example, we compute the generalized chromatic polynomial of the bridge graph depicted in Figure 9. The resulting transformations using the composition order given by (14) are presented in Table 


\begin{tabular}{|c|lll|c|}
\hline Step & Partition & $p$ & $q$ & Count \\
\hline & 0 & 0 & 0 & 1 \\
\hline+1 & 1 & 1 & 0 & 1 \\
\hline+2 & $1 \mid 2$ & 2 & 0 & 1 \\
& $12^{*}$ & 0 & 1 & 1 \\
\hline$\{1,2\}$ & $1 \mid 2$ & 2 & 0 & 1 \\
\hline+3 & $1|2| 3$ & 3 & 0 & 1 \\
& $13^{*} \mid 2$ & 1 & 1 & 1 \\
& $23^{*} \mid 1$ & 1 & 1 & 1 \\
\hline$\{1,3\}$ & $1|2| 3$ & 3 & 0 & 1 \\
& $23^{*} \mid 1$ & 1 & 1 & 1 \\
\hline-1 & $2 \mid 3$ & 3 & 0 & 1 \\
& $23^{*}$ & 1 & 1 & 1 \\
\hline$\{2,3\}$ & $2 \mid 3$ & 3 & 0 & 1 \\
\hline+4 & $2|3| 4$ & 4 & 0 & 1 \\
& $2|3| 4^{*}$ & 2 & 1 & 1 \\
\hline
\end{tabular}

\begin{tabular}{|c|ccc|c|}
\hline Step & Partition & $p$ & $q$ & Count \\
\hline & $24^{*} \mid 3$ & 2 & 1 & 1 \\
& $2 \mid 34^{*}$ & 2 & 1 & 1 \\
\hline$\{2,4\}$ & $2|3| 4$ & 4 & 0 & 1 \\
& $2|3| 4^{*}$ & 2 & 1 & 1 \\
& $2 \mid 34^{*}$ & 2 & 1 & 1 \\
\hline-2 & $3 \mid 4$ & 4 & 0 & 1 \\
& $3 \mid 4^{*}$ & 2 & 1 & 1 \\
& $34^{*}$ & 2 & 1 & 1 \\
\hline$\{3,4\}$ & $3 \mid 4$ & 4 & 0 & 1 \\
& $3 \mid 4^{*}$ & 2 & 1 & 1 \\
\hline-3 & 4 & 4 & 0 & 1 \\
& $4^{*}$ & 2 & 1 & 1 \\
\hline-4 & 0 & 4 & 0 & 1 \\
& $\emptyset$ & 2 & 1 & 1 \\
\hline
\end{tabular}

Tab. 1: Computation of the generalized chromatic polynomial of the graph in Figure 9 .

1. At the end, two states remain corresponding to the coefficients $a_{40}=1$ and $a_{21}=1$. The polynomial in equation (8) is thus $x^{4}+x^{2} y$, which can easily be transformed via Corollary 7 into

$$
P(G ; x, y)=x^{4}-5 x^{2} y+6 x y-4 y+2 y^{2}
$$

A minor modification of the presented algorithm permits the computation of a function $Q^{*}(G ; x, y)$ that is defined analogously to $Q(G ; x, y)$ with the exception that the coefficient $a_{p q}$ counts the number of partitions consisting of $p$ singletons and $q$ other blocks such that each block forms a clique.

The computation of $Q^{*}(G ; x, y)$ again requires a nice path decomposition of $G$. However, the edge insertion step is not necessary. A state $z=(\pi, f)$ consists of a labeled partition $\pi$ as index and a bivariate polynomial $f$ as value. This time, the label of a block of $\pi$ indicates that this block belongs to a clique that contains already deactivated vertices. Such a labeled block can not be extended to a larger clique. The coefficient $\left[x^{p} y^{q}\right] f$ of the polynomial $f$ gives the number of partitions represented by the index $\pi$ that consist of $p$ singletons and $q$ other cliques. The initial state for the empty graph is $(\emptyset, 1)$. We use the following elementary transformations:

Vertex activation $(+v)$ : A new vertex may always form a one-vertex clique. Thus, every state $(\pi, f)$ induces a new state $(\pi \mid v, x f)$. If $v$ is adjacent to a unlabeled singleton $\{w\}$ of $\pi$ then $\{v, w\}$ forms a two-vertex clique of $G$ producing a new state $\left(\pi-\{w\} \mid\{v, w\}, x^{-1} y f\right)$. If $v$ is adjacent to all vertices of a non-singleton block $W$ that is not labeled then an existing clique may be enlarged by $v$ generating a new state $(\pi-W \mid W \cup\{v\}, f)$.

Vertex deactivation $(-v)$ : The vertex $v$ has to be removed from the index (i.e. the labeled partition). If $v$ is removed from a non-singleton block then the remaining set becomes a labeled block. The values remain unchanged. A reduction of the number of states is possible by collecting states with identical index into one state the value of which is the sum of the values of the collection. 
The application of this algorithm to the graph of Figure 9 yields

$$
Q^{*}(G ; x, y)=y^{4}+5 x y^{2}+2 x y+2 x^{2} .
$$

Let $\bar{G}$ be the complement of the graph $G$. It follows from the definition of $Q^{*}$ that $Q(G ; x, y)=$ $Q^{*}(\bar{G} ; x, y)$. This gives the following result:

Theorem 13 The generalized chromatic polynomial can be computed in polynomial time for graphs of restricted pathwidth and for graphs whose complement is of restricted pathwidth. In particular, the matching polynomial, the independence polynomial, and the chromatic polynomial can be computed in polynomial time for graphs of restricted pathwidth and for graphs whose complement is of restricted pathwidth.

\section{Acknowledgement}

The authors wish to express their gratitude to the referees for the many helpful suggestions that resulted in an improvement of the paper. In particular, the derivations of the generalized chromatic polynomial of a path (Subsection 5.3) and a cycle (Subsection 5.4) were greatly simplified by one of the referees, whose solution we adopted with minor changes and whom we especially would like to thank for his worthy contribution.

\section{References}

[1] H.L. Bodlaender: A partial $k$-arboretum of graphs with bounded treewidth, Theoret. Comput. Sci. 209 (1998), 1-45.

[2] T. Brylawski: The broken circuit complex, Trans. Amer. Math. Soc. 234 (1977), 417-433.

[3] T. Brylawski \& J. Oxley: The broken-circuit-complex: its structure and factorizations, Europ. J. Comb. 2 (1981), 107-121.

[4] K. Dohmen: Improved Bonferroni inequalities via union-closed set systems, J. Combin. Theory Ser. A 92 (2000), 61-67.

[5] C. Godsil \& G. Royle: Algebraic Graph Theory, Graduate Texts in Mathematics, 207, SpringerVerlag, New York, 2001.

[6] F. Lazebnik: Some corollaries of a theorem of Whitney on the chromatic polynomial, Discrete Math. 87 (1991), 53-64.

[7] R.C. Read: An introduction to chromatic polynomials, J. Combin. Theory Ser. B 4 (1968), 52-71.

[8] G.-C. Rota: On the Foundations of Combinatorial Theory: I. Theory of Möbius functions, $Z$. Wahrscheinlichkeitstheorie Verw. Gebiete 2 (1964), 340-368.

[9] P. Scheffler: Die Baumweite von Graphen als ein Maß für die Kompliziertheit algorithmischer Probleme, Report R-MATH-04/89, Karl-Weierstraß-Institut für Mathematik, Akademie der Wissenschaften der DDR, Berlin, 1989.

[10] R.P. Stanley: Enumerative Combinatorics, Vol. 1, Wadsworth \& Brooks/Cole, Monterey, Ca., 1986. 
[11] R.P. Stanley: Enumerative Combinatorics, Vol. 2, Cambridge University Press, 1999.

[12] D.M. Thilikos, M.J. Serna, H.L. Bodlaender: Constructive linear time algorithms for small cutwidth and carving-width, Proc. 11th International Symposium on Algorithms and Computation, ISAAC '00, Lecture Notes in Computer Science 1969, Springer Verlag, 2000, pp. 192-203.

[13] W.T. Tutte: Graph Theory, Addison-Wesley, Menlo Park, 1984.

[14] W.T. Tutte: A ring in graph theory, Proc. Cambridge Phil. Soc. 43 (1947), 26-40.

[15] H. Whitney: A logical expansion in mathematics, Bull. Amer. Math. Soc. 38 (1932), 572-579.

[16] H.S. Wilf: Which polynomials are chromatic?, Proc. Colloquio Internazionale sulle Teorie Combinatorie (Roma, 1973), Tomo I, Atti dei Convegni Lincei, No. 17, Accademia Naz. Lincei (Rome), 1976, pp. 247-256. 
